\title{
Low-Glycemic-Index Diet Relieving Migraine but Inducing Muscle Cramps
}

\author{
Josef Finsterer ${ }^{1}$ Marlies Frank ${ }^{2}$ \\ ${ }^{1}$ Department of Neurological, Krankenanstalt Rudolfstiftung, \\ Messerli Institute, Austria \\ ${ }^{2}$ First Medical Department, Krankenanstalt Rudolfstiftung, Vienna, \\ Austria
}

\begin{abstract}
Address for correspondence Josef Finsterer, Krankenanstalt Rudolfstiftung, Messerli Institute, Postfach 20, 1180 Vienna, Austria (e-mail: fifigs1@yahoo.de).
\end{abstract}

J Neurosci Rural Pract 2019;10:552-554

\section{Introduction}

Low-glycemic-index diet (LGID, the Atkins diet) and its modifications are characterized by daily intake of a high fraction of fat and a low fraction of carbohydrates. The Atkins diet has been repeatedly shown to exhibit a beneficial effect on various neurological conditions. LGID has been shown to be particularly effective in various types of mitochondrial epilepsy, ${ }^{1}$ exercise-induced dyskinesias, ${ }^{2}$ writer's cramp, ${ }^{2}$ in migraine, ${ }^{3}$ and mitochondrial myopathy of mice. ${ }^{4}$ Here, we report a patient with migraine who significantly profited from the LGID but also experienced side effects.

\section{Case Report}

The patient is a 57 -year-old female, height $175 \mathrm{~cm}$, weight $68 \mathrm{~kg}$, who developed classical migraine at 14 years of age. Occasionally, migraine was preceded by aura and was previously associated with severe nausea and vomiting. Without taking any medication, migraine attacks lasted 24 hours to 5 days. Aura had become less frequent during the last years and manifested without headache meanwhile. Migraine occurred with a frequency of up to 10 (8-12) attacks per month since 20 years, along with marked changes of the weather as a triggering factor. The patient also experienced episodes of status migrainosus lasting up to 5 days. Initially, migraine was treated with various nonsteroidal analgesic drugs, sumatriptan, and as a prophylaxis, $\beta$-blockers, and topiramate with side effects. Since 10 years, the patient was taking zolmitriptan in the form of a nasal spray, metoclopramide, and domperidon with a beneficial effect. Since adolescence she also experienced polyarthralgia. At the age of 42 years, spondylarthritis with permanent enthesitis and bursitis with antibodies against HLAb27 was diagnosed why hydroxychloroquine and naproxen were prescribed. Since 55 years of age, the patient experienced permanent hypoesthesias of the feet and transient paresthesias over the right calf or around the left lower leg, several times per week. Since years, she also experienced sudden-onset attacks of fatigue, particularly in the afternoon but sometimes also after getting up in the morning. Since the age of 56 years, she suffered from a medial epicondylitis on the right side. At the 
age of 56 years, she underwent discectomy L5/S1 because of a disc prolapse. Her history was additionally positive for lactose and fructose intolerance since at least 20 years with chronic diarrhea since 10 years. Her family history was positive for migraine (father), stroke (grandmother from the mother's side, grand-grandmother from the mother's side), gibbus (grandmother from the mother's side), and carcinoma (father).

At 57 years of age, she first started with a strict LGID, comprising $70 \%$ fat, $20 \%$ protein, and $10 \%$ carbohydrates. Within a few days, she recognized a reduction of intensity and frequency of migraine attacks by two-thirds compared with the status before starting the diet. During the first 12 weeks under LGID, she experienced only three migraine attacks, which were always associated with a weather change. Furthermore, manifestations of fructose and lactose intolerance markedly improved and attacks of sudden fatigue during the day and epicondylitis completely resolved since initiation of the LGID. Eight weeks after starting the LGID, she suddenly experienced extremely intense muscle cramps of the left calve visual analogue scale (VAS 8), which radiated to the thighs, buttocks, and from there to the right leg. She attributed the muscle cramps to LGID, although she had experienced occasional cramps of the right calve already since 1.5 years before LGID. Muscle cramps had started even before as mild carpopedal spasms bilaterally since the age of 54 years. The intensity of muscle cramps increased after she had been physically active during the day. Muscle cramps could be relieved by sitting. Eight weeks after starting LGID, she first recognized fasciculations of the buttocks and right calve. She also experienced spontaneous myalgias, which she described as permanent sore muscles with a maximum in the morning. Nerve conduction studies of the left-ulnar nerve, right-peroneal nerve, right-tibial nerve, and right-sural nerve were all normal. Since L-carnitine and magnesium did not relieve muscle cramps, she slightly reduced the amount of fat and increased the carbohydrate portion with some improvement. To further resolve muscle cramps, she took steroids thrice, novaminsulfon and wobenzym, in addition to her drugs against migraine and spondylarthritis, with a beneficial effect.

\section{Discussion}

The presented patient is interesting since she experienced marked relief from long-term migraine after application of the LGID. Not only did the frequency of migraine attacks decline from 10 /month to 1 /month but also the intensity of the attacks. A beneficial effect of LGID for migraine has been previously reported in an 8-year-old male with glucose transporter-1 deficiency due to a mutation in SLC2A1. ${ }^{5}$ Hemiplegic migraine permanently resolved on adherence to a modified LGID. ${ }^{5}$ In a study of 45 migraine patients on LGID during 6 months, significant improvement could be achieved compared with the control group. In twins aged 5 years and 10 years, respectively, both recovered almost completely under LGID from exercise-induced dyskinesia, writer's cramp, and migraine with aura. ${ }^{2} \mathrm{~A}$ beneficial effect of the Atkins diet on migraine has been first shown in 1928 by Schnabel et al.

LGID is not only beneficial for migraine but also for mitochondrial epilepsy, writer's cramp, exercise-induced dyskinesia, ${ }^{2}$ and mitochondrial myopathy in mice. ${ }^{4}$ The improvement of epilepsy, following fasting, is known since biblical times but has been first described by Guelpa and Marie in 1911. The effect was confirmed by a report by Geyelin in 1921. In a recent study of nine patients with refractory epilepsy, however, LGID was effective in only two of them. The reason why LGID is effective in these disorders remains speculative, but it can be assumed that it is attributable to the ability of ketone bodies to enhance the mitochondrial energy metabolism and to counteract neuronal inflammation. It has been also hypothesized that ketone bodies could exhibit an inhibitory effect on neuronal ion channels, reduce neuronal excitability by decreasing the cerebral $\mathrm{pH}$, reduce cortical spreading depression propagation, or shift the amino acid metabolism such that more inhibitory transmitters are produced. There are, however, also reports showing that LGID may worsen genetic epilepsy due to a mutation in the SLC13A5 gene. LGID may also exhibit an immune-enhancing effect in patients with malignant gliomas.

A further interesting point of the presented case is that LGID-enhanced muscle cramps. An argument in favor of the LGID as the trigger of muscle cramps is that steroids, which increase blood glucose, or reduction of the fat and increase of the carbohydrate-portion of the LGID relieved muscle cramps. LGID also improved myalgias. Although the cause of muscle cramps and sensory disturbances remains unknown, it can be speculated that together with migraine, they may have a common cause, such as a mitochondrial defect. Discontinuation of chloroquine relieved myalgias and muscle cramps. Vertebral stenosis, disc prolapse, arteriopathy, or venous thrombosis were excluded as causes of the cramping.

It is concluded that LGID may significantly reduce intensity and duration of migraine attacks. Most likely, this effect occurs only in patients in whom migraine is due to a mitochondrial defect. In case LGID is effective, defective glucose metabolism is quite likely.

\section{Funding}

None.

\section{Conflict of Interest}

None declared.

\section{References}

1 Cervenka MC, Henry BJ, Felton EA, Patton K, Kossoff EH. Establishing an adult epilepsy diet center: Experience, efficacy and challenges. Epilepsy Behav 2016;58:61-68

2 Di Lorenzo C, Coppola G, Bracaglia M, et al. Cortical functional correlates of responsiveness to short-lasting preventive intervention with ketogenic diet in migraine: a multimodal evoked potentials study. J Headache Pain 2016;17:58

3 Barbanti P, Fofi L, Aurilia C, Egeo G, Caprio M. Ketogenic diet in migraine: rationale, findings and perspectives. Neurol Sci 2017;38(Suppl 1):111-115 
4 Ahola-Erkkilä S, Carroll CJ, Peltola-Mjösund K, et al. Ketogenic diet slows down mitochondrial myopathy progression in mice. Hum Mol Genet 2010;19(10):1974-1984
5 Di Lorenzo C, Coppola G, Sirianni G, et al. Migraine improvement during short lasting ketogenesis: a proof-of-concept study. Eur J Neurol 2015;22(1):170-177 